\title{
The association between acute fatty liver disease and nitric oxide during malaria in pregnancy
}

Mamoru Niikura $^{1 *} \mathbb{0}$, Toshiyuki Fukutomi ${ }^{2}$, Shoichiro Mineo ${ }^{3}$, Jiro Mitobe ${ }^{1}$ and Fumie Kobayashi ${ }^{4}$

\begin{abstract}
Background: Liver disease is a common feature of malaria in pregnancy, but its pathogenesis remains unclear.

Methods: To understand the pathogenesis of liver disease during malaria in pregnancy, comparative proteomic analysis of the liver in a mouse model of malaria in pregnancy was performed.

Results: Decreased levels of mitochondrial and peroxisomal proteins were observed in the livers of pregnant mice infected with the lethal rodent malaria parasite Plasmodium berghei strain NK65. By contrast, increased levels of perilipin-2, amyloid A-1, and interferon (IFN)- $\gamma$ signalling pathway-related proteins were observed in the livers of infected pregnant mice, suggesting that IFN- $\gamma$ signalling may contribute to the development of liver disease during malaria in pregnancy. IFN- $\gamma$ signalling is a potential trigger of inducible nitric oxide synthase (iNOS) expression. Liver disease associated with microvesicular fatty infiltration and elevated liver enzymes in pregnant wild-type mice infected with malaria parasites was improved by iNOS deficiency.

Conclusions: In this study, a causative role of iNOS in liver disease associated with microvesicular fatty infiltration during malaria in pregnancy was demonstrated. These findings provide important insight for understanding the role of iNOS-mediated metabolic responses and the pathogenesis of high-risk liver diseases in pregnancy, such as acute fatty liver.
\end{abstract}

Keywords: Fatty liver, iNOS, Liver disease, Malaria, Pregnancy, Proteome

\section{Background}

Individuals living in malaria-endemic regions acquire protective immunity against malaria parasites and often show asymptomatic infection. However, women are highly susceptible to malaria infection because of low immunity during pregnancy $[1,2]$. Malaria during pregnancy, in addition to maternal illness or death, is implicated in placental malaria, which has been correlated with adverse pregnancy outcomes [3-5]. Malaria in

*Correspondence: mniikura@ks.kyorin-u.ac.jp

${ }^{1}$ Department of Infectious Diseases, Kyorin University School of Medicine, 6-20-2 Shinkawa, Mitaka, Tokyo 181-8611, Japan

Full list of author information is available at the end of the article pregnancy is a major public health problem in endemic areas, especially in Africa [6].

Severe anaemia, thrombocytopenia, and liver dysfunction are common features of severe malaria [7]. These pathological features of pregnant women infected with Plasmodium falciparum can be confused with haemolytic anaemia, elevated liver enzymes, and low platelet count (HELLP) syndrome [8-10]. Similar to HELLP syndrome, pregnant women infected with malaria parasites undergo spontaneous abortion, stillbirth, and premature delivery [3-5]. In North-western Colombia, hepatic dysfunction is the most frequent complication in patients with severe cases ( 9 of 15 patients) [11]. These findings indicate that hepatic dysfunction is common during human placental malaria.

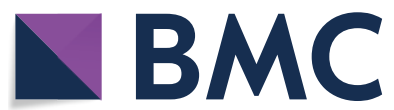

(c) The Author(s) 2021. Open Access This article is licensed under a Creative Commons Attribution 4.0 International License, which permits use, sharing, adaptation, distribution and reproduction in any medium or format, as long as you give appropriate credit to the original author(s) and the source, provide a link to the Creative Commons licence, and indicate if changes were made. The images or other third party material in this article are included in the article's Creative Commons licence, unless indicated otherwise in a credit line to the material. If material is not included in the article's Creative Commons licence and your intended use is not permitted by statutory regulation or exceeds the permitted use, you will need to obtain permission directly from the copyright holder. To view a copy of this licence, visit http://creativecommons.org/licenses/by/4.0/. The Creative Commons Public Domain Dedication waiver (http://creativeco mmons.org/publicdomain/zero/1.0/) applies to the data made available in this article, unless otherwise stated in a credit line to the data. 
In a study using a mouse model of malaria in pregnancy, when mice immunized with the attenuated malaria parasite Plasmodium berghei strain XAT were infected with the lethal malaria parasite P. berghei strain NK65, they showed severe liver disease and placental pathology in the late phase of pregnancy, resulting in adverse pregnancy outcomes [12]. Histological analysis revealed microvesicular fatty infiltration in livers obtained from the mouse model of malaria in pregnancy. Liver disease associated with the deposition of fat showed pathological features similar to those of acute fatty liver of pregnancy (AFLP).

Differentiation of HELLP syndrome and AFLP can be challenging, because the initial pathological features of these diseases are similar [13]. It is believed that defects in fatty acid metabolism during pregnancy are involved in acute fatty liver development [14]. Indeed, approximately $20 \%$ of cases of AFLP have been associated with long-chain 3-hydroxyacyl CoA dehydrogenase deficiency $[15,16]$. However, to date, the detailed mechanisms by which pregnant women develop HELLP syndrome and AFLP are not fully understood.

The study of a mouse model exhibiting liver disease associated with microvesicular fatty infiltration during malaria in pregnancy may help improve our understanding of the pathogenesis of liver diseases in pregnancy, such as AFLP. To investigate the pathogenesis of acute fatty liver disease in pregnancy, a comparative proteomic analysis of the liver in a mouse model of malaria in pregnancy was performed. The effects of inducible nitric oxide synthase (iNOS) deficiency on acute fatty liver disease during malaria in pregnancy were also investigated using the mouse model.

\section{Methods}

\section{Animals and ethics}

Female and male C57BL/6 J (B6) mice (5-6 weeks of age) were purchased from CLEA Japan Inc. (Tokyo, Japan). iNOS-deficient mice [17] were a gift from Dr. Takuya Sakurai (Department of Molecular Predictive Medicine and Sport Science, Kyorin University, School of Medicine). The experiments were approved (\#220) by the Experimental Animal Ethics Committee of Kyorin University School of Medicine. All experimental animals were maintained in the animal facility in a specific pathogen-free unit with sterile bedding, food, and water. A female mouse $>9-11$ weeks of age was mated for 1 day with a male mouse in a cage and examined for the presence of a vaginal plug the next morning.

The infection studies included frequent observations to determine humane endpoints, such as an inability to ambulate sufficiently to obtain water or food. At the indicated time points, mice were euthanized by cervical dislocation under isoflurane or pentobarbital sodium anaesthesia. All experiments were designed to minimize animal suffering. When illness or death was expected due to experimental infections, mice were visually checked by investigators at least twice daily (including weekends and holidays). The investigators who conducted the experiments had completed the Experimental Animal Ethics Committee training course on animal care and handling. All experiments were performed in accordance with the guidelines and regulations of animal experimentation at Kyorin University. All animal experiments were also carried out in compliance with the ARRIVE guidelines.

\section{Parasites and infections}

Lethal $P$. berghei NK65 and attenuated P. berghei XAT parasites were stored as frozen stocks in liquid nitrogen $[12,18]$. Malaria parasite-infected erythrocytes were generated in donor mice inoculated intraperitoneally with a frozen stock of parasite. The donor mice were monitored for parasitaemia daily and bled for experimental infection during periods of ascending parasitaemia. Female mice 5-6 weeks of age were injected intravenously with $1 \times 10^{4}$ erythrocytes infected with attenuated parasites. On day 29 post-infection, these female mice were mated with a $>9$-week-old male mouse for 1 day and examined for the presence of a vaginal plug the following morning (immunized (IM) pregnant and non-pregnant mice). Mice immunized with attenuated parasites with or without a vaginal plug were infected intravenously with $1 \times 10^{4}$ erythrocytes infected with lethal parasites (IM pregnant and non-pregnant mice infected with $P$. berghei NK65).

\section{Parasitaemia}

Methanol-fixed tail blood smears stained with 3\% Giemsa diluted with phosphate buffer ( $\mathrm{pH}$ 7.2) for $45 \mathrm{~min}$ were observed under a microscope. The number of infected erythrocytes in 250 erythrocytes was counted when parasitaemia exceeded $10 \%$, and $1 \times 10^{4}$ erythrocytes were examined in mice with milder parasitaemia. The percentage of parasitaemia was calculated as follows: [(number of infected erythrocytes)/(total number of erythrocytes) $] \times 100$.

\section{Histological examination and measurement of parameters of liver injury}

Livers and blood were obtained from uninfected pregnant and non-pregnant mice, and IM pregnant and nonpregnant mice infected with $P$. berghei NK65, on day 17 post-mating. Note that "uninfected mice" were neither immunized with $P$. berghei XAT nor infected with $P$. berghei NK65. Placentas and fetuses were obtained from uninfected pregnant mice, IM pregnant mice, and IM 
pregnant mice infected with $P$. berghei NK65 on day 17 post-mating. Tissues and fetuses were fixed in $10 \%$ buffered formalin and embedded in paraffin, or fixed in $4 \%$ paraformaldehyde and frozen at $-80{ }^{\circ} \mathrm{C}$. Sections $(6 \mu \mathrm{M}$ thick) were stained with haematoxylin and eosin or Sudan IV. The latter sections were photographed at $400 \times$ magnification using an All-in-one Fluorescence Microscope (BZ-9000; KEYENCE Japan, Osaka, Japan). Fat droplets in the photographs were counted using BZ-II Analyzer software (KEYENCE Japan). Blood was centrifuged at $500 \times g$ for $10 \mathrm{~min}$. The resulting supernatants were stored at $-20^{\circ} \mathrm{C}$ and used as plasma. The levels of aspartic aminotransferase, alanine aminotransferase, and glucose in plasma were determined at Nagahama Life Science Laboratory (Shiga, Japan).

\section{Comparative proteomic analysis}

Livers were obtained from uninfected pregnant and nonpregnant mice, and IM pregnant and non-pregnant mice infected with $P$. berghei NK65 on day 17 post-mating. Proteins were extracted using the Mammalian Protein Extraction Reagent (Thermo Fisher Scientific, Waltham, MA, USA) according to the manufacturer's protocol and treated with trypsin. Comparative proteomic analyses were performed as previously described $[19,20]$. The fractionated peptides were injected into a trap column (C18, $0.3 \times 5 \mathrm{~mm}$; L-column, Chemicals Evaluation and Research Institute, Tokyo, Japan) and an analytical column $(\mathrm{C} 18,0.075 \times 120 \mathrm{~mm}$; Nikkyo Technos, Tokyo, Japan) attached to a nano liquid chromatography-tandem mass spectrometry (nanoLC-MS/MS) system. The nanoLC-MS/MS analysis was conducted using an LTQ Orbitrap Velos mass spectrometer (Thermo Fisher Scientific) equipped with a nanoLC interface (KYA, Tokyo, Japan) and a nano high-performance liquid chromatography (nanoHPLC) system (DiNa; KYA). Purified peptides from the nanoLC were introduced into the LTQ Orbitrap Velos, a hybrid ion-trap Fourier transform mass spectrometer. Full MS and MS/MS scans were followed by higher-energy collisionally activated dissociation. The database search engines Proteome Discoverer 1.4 (Thermo Fisher Scientific) and MASCOT 2.6 (Matrix Science) were used to identify and quantify proteins from the MS, MS/MS, and reporter ion spectra of peptides.

Peptide mass data were matched by searching the NCBInr database. The false discovery rate (FDR) [21] was calculated via peptide sequence analysis using Percolator [22]. High-confidence peptide identifications were obtained by setting a target FDR threshold of $\leq 1.0 \%$ at the peptide level. Proteins showing one or two peptide spectral matches (PSMs) were excluded. Protein levels were normalized to actin, cytoplasmic 1 (accession: P60710), as previously described [23]. The normalized experimental signal was calculated as follows: (observed experimental signal $) \div($ normalisation factor $)$.

Enzyme-linked immunosorbent assay (ELISA) of cytokines An ELISA for the detection of IFN- $\gamma$ or IL-10 in plasma was carried out as described previously [18]. A rat antimouse IFN- $\gamma$ mAb (clone R4-6A2; eBioscience, San Diego, CA, USA) and a rat anti-mouse IL-10 (clone JES5-16E3; eBioscience) were used as the capture antibodies, and a biotinylated, rat anti-mouse IFN- $\gamma$ mAb (clone XMG1.2; eBioscience) and rat anti-mouse IL-10 mAb (clone JES5-2A5; eBioscience) as the detecting antibodies. The concentrations of cytokines in plasma were calculated from standard curves prepared using known quantities of murine recombinant IFN- $\gamma$ (Genzyme, Boston, MA, USA) and IL-10 (Pierce, Rockford, IL, USA). The reaction was visualized using peroxidase-conjugated streptavidin (Zymed) and the substrate 2,2'-azino-bis(3etylbenzthiazoline-6-sulphonic acid) (Wako, Osaka, Japan).

\section{Statistical analysis}

For time-series comparisons, Student's $t$-test and oneand two-way analyses of variance with Fisher's protected least significant difference post-hoc test (Tukey-Kramer and Dunnett test) were performed using the Statcel program (OMS, Saitama, Japan). $P$-values $<0.05$ were considered statistically significant.

\section{Results}

Proteomic analysis of the liver of immunized pregnant mice infected with $P$. berghei NK65

Plasmodium berghei XAT is an attenuated parasite and C57BL/6 mice cured of $P$. berghei XAT infection have acquired protective immunity that suppresses the severe pathology caused by the lethal malaria parasite $P$. berghei NK65 [24]. Mice immunized with the attenuated parasite with or without a vaginal plug were secondarily infected with the lethal malaria parasite $P$. berghei NK65, as described previously [12] (Fig. 1A).

To assess the proteome of the liver of IM pregnant C57BL/6 mice infected with P. berghei NK65, comparative proteomic analysis was performed using proteins extracted from the livers of unpregnant mice challenged with $P$. berghei NK65 and those of pregnant mice challenged with P. berghei NK65 (Fig. 1 and Tables 1, 2, and Additional file 1: Table S1). To investigate the effect of challenge infection on the proteomic alterations of liver during pregnancy, proteins extracted from the livers of unimmunized non-pregnant mice without infection (naïve virgin mice) and unimmunized pregnant mice without infection (naïve pregnant mice) were also analysed (Fig. 1 and Tables 1, 2, and Additional file 1: 

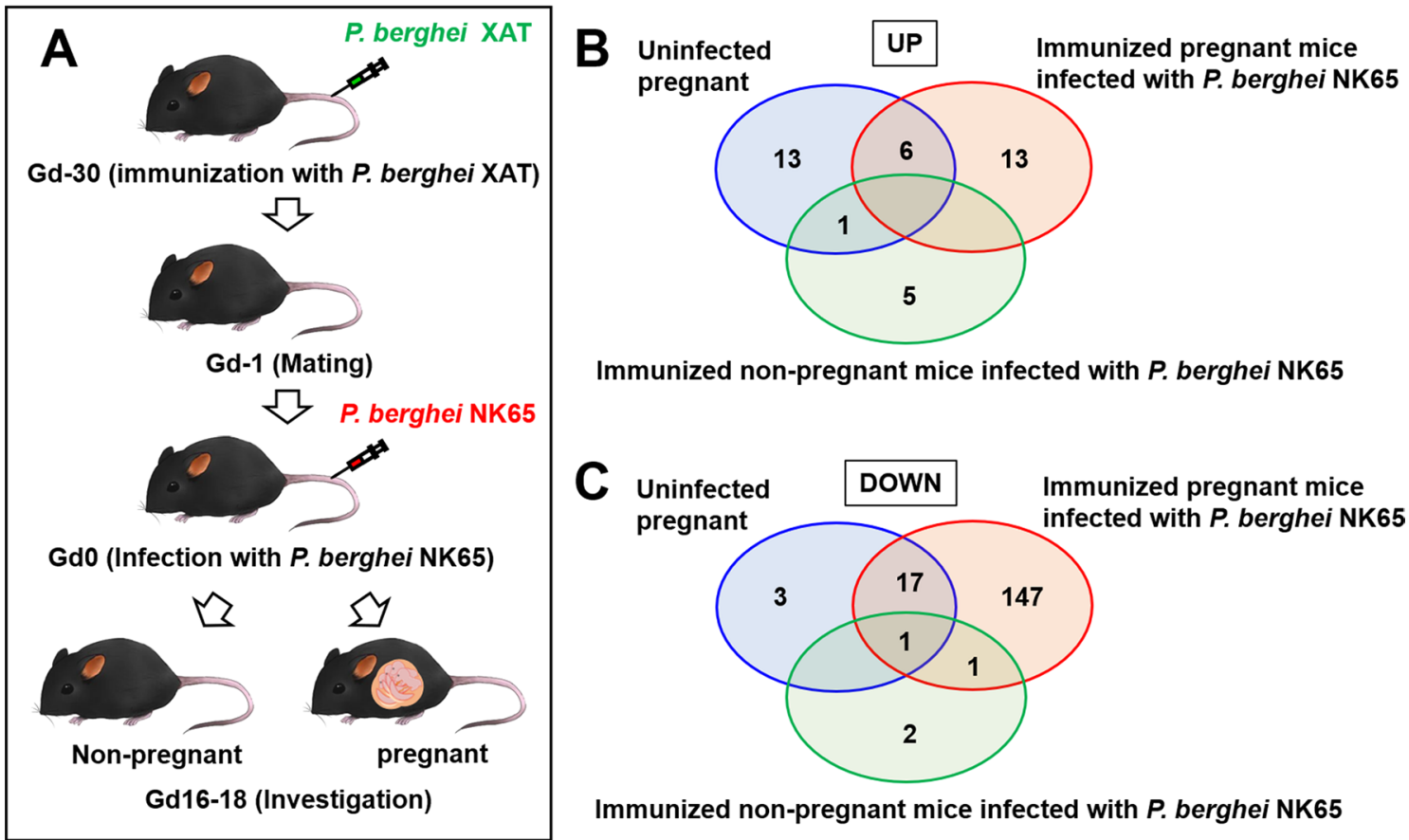

Immunized non-pregnant mice infected with P. berghei NK65

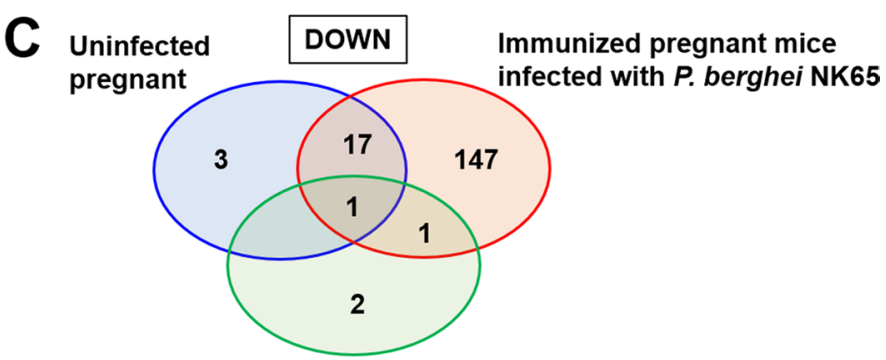

Immunized non-pregnant mice infected with P. berghei NK65

Fig. 1 Proteomic analysis of the liver of immunized pregnant mice infected with malaria parasites. A Schematic representation of immunisation, mating, and infection. Female C57BL/6 (B6) mice were injected with $1 \times 10^{4}$ erythrocytes infected with Plasmodium berghei XAT. Female mice were mated for 1 day and examined for the presence of a vaginal plug the following morning. Immunized mice with or without a vaginal plug were infected with $1 \times 10^{4}$ erythrocytes infected with lethal P. berghei NK65. B, C Venn diagrams of protein levels in the livers of unimmunized pregnant mice without infection (naïve pregnant mice), immunized pregnant mice infected with $P$. berghei NK65, and non-pregnant mice infected with $P$. berghei NK65 that changed twofold (B) or 0.5-fold (C) compared with uninfected non-pregnant mice. Data are representative of three independent experiments

Table S1). A total of 1,758 proteins were detected, among which 1,159 proteins with three or more PSMs were analysed. Protein levels in the liver were normalized to actin, cytoplasmic 1 (accession: P60710) (Additional file 1: Table S1).

The levels of 13 proteins, including perilipin-2, which surrounds the lipid droplet [25], were significantly higher in IM pregnant mice infected with $P$. berghei NK65 than in naïve virgin mice (Fig. $1 \mathrm{~B}$ and Table 1). These results are consistent with our previous findings showing that IM pregnant mice infected with $P$. berghei NK65 develop liver disease associated with microvesicular fatty infiltration [12]. Moreover, serum amyloid A-1 protein (Saa1) and interferon (IFN)- $\gamma$ signalling pathway-related proteins, such as signal transducer and activator of transcription 1 (Stat1), IFN-inducible GTPase 1 (Iigp1), IFN-induced very large GTPase 1 (Gvin1), and IFN-induced protein with tetratricopeptide repeats 3 (Ifit3), were significantly higher in IM pregnant mice infected with $P$. berghei NK65 than in naïve virgin mice and immunized non-pregnant mice infected with $P$. berghei NK65 (Table 1). These results indicate that IFN- $\gamma$ signalling contributes to the development of liver dysfunction during malaria in pregnancy.

By contrast, the levels of 147 proteins in IM pregnant mice infected with $P$. berghei NK65 were markedly decreased compared with naïve virgin mice (Fig. 1C and Additional file 1: Table S1). Of the 147 proteins, the levels of 18 proteins, including mitochondrial atypical kinase (ADCK3), peroxisomal 3-ketoacyl-CoA thiolase $\mathrm{B}$ (Acaa1b), and cytochrome $\mathrm{P} 450$ proteins, were significantly lower in IM pregnant mice infected with $P$. berghei NK65 than in naïve virgin mice (Table 2), indicating liver dysfunction.

\section{Effect of iNOS deficiency on microvesicular fatty infiltration in the liver}

In previous study, the levels of IFN- $\gamma$ and nitric oxide were increased in plasma from IM pregnant mice infected with $P$. berghei NK65 [12]. IFN- $\gamma$ signalling triggers iNOS expression [26], and the free radical nitric oxide produced by iNOS has been implicated in fatty liver [27-29]. To investigate whether nitric oxide produced by iNOS is associated with microvesicular fatty infiltration, female wild-type (WT) and iNOS knockout 
Table 1 Upregulated proteins in the liver of immunized pregnant mice infected with P. berghei NK65

\begin{tabular}{|c|c|c|c|c|c|}
\hline Description & Abbreviation & $\begin{array}{l}\text { Uninfected } \\
\text { pregnant } \\
\text { mouse }\end{array}$ & $\begin{array}{l}\text { Immunized non-pregnant } \\
\text { mouse infected with P. berghei } \\
\text { NK65 }\end{array}$ & $\begin{array}{l}\text { Immunized pregnant mouse } \\
\text { infected with P. berghei NK65 }\end{array}$ & Accession \\
\hline Perilipin-2 OS & Plin2 & 1.14 & 0.84 & 2.13 & P43883 \\
\hline Pyruvate kinase PKM OS & Pkm & 0.85 & 1.12 & 1.73 & P52480* \\
\hline $\begin{array}{l}\text { Nicotinamide N-methyltransferase } \\
\text { OS }\end{array}$ & $\mathrm{Nnmt}$ & 0.52 & 0.57 & 1.13 & O55239* \\
\hline Interferon-inducible GTPase 1 OS & ligp1 & 1.02 & 1.24 & 2.35 & Q9QZ85* \\
\hline Ferritin light chain 1 OS & Ftl1 & 0.33 & 1.61 & 0.79 & P29391* \\
\hline $\begin{array}{l}\text { UMP-CMP kinase 2, mitochondrial } \\
\text { OS }\end{array}$ & Cmpk2 & 1.01 & 1.02 & 2.45 & Q3U5Q7* \\
\hline Haptoglobin OS & $\mathrm{Hp}$ & 1.49 & 0.82 & 4.09 & Q61646* \\
\hline Thymosin beta-10 OS & Tmsb10 & 0.63 & 3.58 & 1.82 & Q6ZWY8* \\
\hline Histone $\mathrm{H} 2 \mathrm{~A}$ type $1-\mathrm{H}$ OS & Hist1h2ah & 0.17 & 0.56 & 0.54 & Q8CGP6* \\
\hline Hemopexin OS & $\mathrm{Hpx}$ & 0.85 & 0.88 & 2.67 & Q91X72* \\
\hline $\begin{array}{l}\text { Signal transducer and activator of } \\
\text { transcription } 1 \text { OS }\end{array}$ & Stat1 & 0.89 & 1.35 & 2.93 & $\mathrm{P} 42225^{*}$ \\
\hline Z-DNA-binding protein 1 OS & Zbp1 & 1.16 & 1.30 & 3.91 & Q9QY24* \\
\hline Coronin-1A OS & Corola & 0.58 & 1.28 & 2.03 & O89053* \\
\hline Metallothionein-2 OS & Mt2 & 2.17 & 0.22 & 8.34 & P02798* \\
\hline Metallothionein-1 OS & Mt1 & 1.38 & 0.61 & 6.02 & P02802* \\
\hline $\begin{array}{l}\text { Interferon-induced very large } \\
\text { GTPase } 1 \text { OS }\end{array}$ & Gvin1 & 0.79 & 1.66 & 4.84 & Q80SU7* \\
\hline $\begin{array}{l}\text { Interferon-induced protein with } \\
\text { tetratricopeptide repeats } 3 \text { OS }\end{array}$ & Ifit3 & 0.54 & 1.41 & 9.25 & Q64345* \\
\hline Serum amyloid A-1 protein OS & Saa1 & 0.72 & 0.71 & 16.41 & P05366* \\
\hline
\end{tabular}

Protein levels in the livers of immunized pregnant mice infected with P. berghei NK65 were compared with the levels in uninfected non-pregnant mice. Proteins with at least three peptide spectral matches and a fold change $>2.0$ compared with unimmunized non-pregnant mice without infection (naïve virgin mice) are listed. Asterisks indicate proteins with significantly higher levels than in unimmunized non-pregnant mice without infection (naïve virgin mice) (>twofold change). Additional file 1: Table S1 shows all proteins detected in this study. Data are representative of three independent experiments

(KO) mice immunized with the attenuated parasite $P$. berghei XAT, with or without a vaginal plug, were infected with $P$. berghei NK65. After infection with $P$. berghei NK65, immunized non-pregnant WT and iNOSKO mice showed low levels of parasitaemia and were cured spontaneously (Fig. 2A). By contrast, IM pregnant iNOS-KO mice infected with $P$. berghei NK65 showed a rapid increase in parasitaemia (Fig. 2A). The course of parasitaemia in IM pregnant iNOS-KO mice infected with $P$. berghei NK65 was similar to that of IM pregnant WT mice infected with $P$. berghei NK65 (Fig. 2A). However, histological changes, such as microvesicular fatty infiltration, observed in the liver of IM pregnant WT mice infected with $P$. berghei NK65 were improved in IM pregnant iNOS-KO mice infected with $P$. berghei NK65 on day 17 post-mating (Fig. 2B-G).

Increased levels of liver enzymes, such as aspartic aminotransferase, in IM pregnant WT mice infected with $P$. berghei NK65 on day 17 post-mating were significantly decreased in IM pregnant iNOS-KO mice infected with P. berghei NK65 (Fig. 3A, B). By contrast, levels of glucose in IM pregnant WT and iNOS-KO mice infected with
P. berghei NK65 on day 17 post-mating were lower than those in immunized non-pregnant mice infected with $P$. berghei NK65 (Fig. 3C). These results suggest that nitric oxide produced by iNOS is associated with microvesicular fatty infiltration.

Unimmunized C57BL/6 mice infected with the lethal malaria parasite $P$. berghei NK65 exhibit increased parasitaemia in the early phase of infection and suffer from liver injury; all mice die within 2 weeks post-infection [30]. To investigate whether liver injury caused by iNOSderived nitric oxide develops in unimmunized pregnant mice infected with $P$. berghei NK65, unimmunized pregnant WT and iNOS-KO mice on day 12 post-mating were infected with $P$. berghei NK65. Parasitaemia in unimmunized pregnant mice infected with $P$. berghei NK65 rapidly increased compared with unimmunized non-pregnant mice infected with $P$. berghei NK65 (Additional file 2: Fig. S1). This course of parasitaemia was not altered by iNOS deficiency (Additional file 2: Fig. S1). Histological analysis revealed that liver disease observed in infected non-pregnant and pregnant iNOS-KO mice was similar to that in infected WT mice (Additional 
Table 2 Downregulated proteins in the liver of immunized pregnant mice infected with P. berghei NK65

\begin{tabular}{|c|c|c|c|c|c|}
\hline Description & Abbreviation & $\begin{array}{l}\text { Uninfected } \\
\text { pregnant } \\
\text { mouse }\end{array}$ & $\begin{array}{l}\text { Immunized non-pregnant } \\
\text { mouse infected with P. berghei } \\
\text { NK65 }\end{array}$ & $\begin{array}{l}\text { Immunized pregnant mouse } \\
\text { infected with P. berghei NK65 }\end{array}$ & Accession \\
\hline Major urinary protein 2 OS & Mup2 & 2.28 & 2.27 & 0.45 & P11589† \\
\hline Cytochrome P450 2C70 OS & Cyp2c70 & 1.99 & 0.97 & 0.47 & Q91W64t \\
\hline $\begin{array}{l}\text { Atypical kinase ADCK3, mitochon- } \\
\text { drial OS }\end{array}$ & Adck3 & 0.62 & 0.97 & 0.15 & Q60936† \\
\hline Cytochrome P450 2A4 OS & Cyp2a4 & 1.77 & 0.29 & 0.44 & P15392† \\
\hline Pericentrin OS & Pent & 0.84 & 0.67 & 0.23 & P48725† \\
\hline Fatty acid desaturase 2 OS & Fads2 & 1.53 & 0.82 & 0.45 & Q9Z0R9† \\
\hline Cytochrome P450 2C54 OS & Cyp2c54 & 0.79 & 1.11 & 0.26 & Q6XVG2† \\
\hline $\begin{array}{l}\text { Guanidinoacetate N-methyltrans- } \\
\text { ferase OS }\end{array}$ & Gamt & 1.41 & 1.05 & 0.47 & O35969† \\
\hline $\begin{array}{l}\text { 3-ketoacyl-CoA thiolase B, peroxi- } \\
\text { somal OS }\end{array}$ & Acaalb & 0.66 & 0.86 & 0.22 & Q8VCH0† \\
\hline Corticosteroid-binding globulin OS & Serpina6 & 8.48 & 0.21 & 2.84 & Q06770† \\
\hline Cytochrome P450 2C29 OS & Cyp2c29 & 1.15 & 1.24 & 0.39 & Q64458† \\
\hline D-dopachrome decarboxylase OS & Ddt & 0.89 & 1.15 & 0.30 & O35215† \\
\hline Proteasome subunit beta type- 5 OS & Psmb5 & 1.08 & 0.76 & 0.37 & O55234† \\
\hline $\begin{array}{l}6.8 \mathrm{kDa} \text { mitochondrial proteolipid } \\
\text { OS }\end{array}$ & Mp68 & 0.88 & 0.82 & 0.31 & P56379† \\
\hline Apolipoprotein C-III OS & Apoc3 & 2.03 & 1.10 & 0.77 & P33622† \\
\hline Phenylalanine-4-hydroxylase OS & Pah & 0.89 & 0.84 & 0.35 & P16331† \\
\hline Fatty acid synthase OS & Fasn & 1.31 & 1.70 & 0.51 & P19096† \\
\hline $\begin{array}{l}\text { Dimethylaniline monooxygenase } \\
\text { [N-oxide-forming] } 3 \text { OS }\end{array}$ & Fmo3 & 2.31 & 1.41 & 0.92 & P97501† \\
\hline
\end{tabular}

Protein levels in the livers of immunized pregnant mice infected with P. berghei NK65 were compared with levels in uninfected non-pregnant mice. Proteins with at least three peptide spectral matches and fold change $<0.5$ compared with unimmunized non-pregnant mice without infection (naïve virgin mice) are listed. Daggers indicate proteins with significantly lower levels than in unimmunized non-pregnant mice without infection (naïve virgin mice) $(<0.4$-fold change). Additional file 1: Table S1 shows all proteins detected in this study. Data are representative of three independent experiments

file 2: Fig. S1). These results suggest that the impact of nitric oxide produced by iNOS is different in unimmunized and immunized mice.

\section{Effect of iNOS deficiency on the proteome in liver dysfunction}

To investigate the effect of iNOS deficiency on the proteome of damaged livers of IM pregnant WT mice infected with $P$. berghei NK65, comparative proteomic analysis was performed (Tables 3, 4, and Additional file 3: Table S2). In the comparative proteomics analysis, 1,716 proteins were detected; of these, 1,154 proteins with three or more PSMs were analysed. Protein levels were normalized to actin, cytoplasmic 1 (accession: P60710) (Additional file 3: Table S2).

The levels of 18 proteins, including perilipin-2, were markedly decreased in IM pregnant iNOS-KO mice infected with $P$. berghei NK65 compared with IM pregnant WT mice infected with $P$. berghei NK65 (Table 3). These results support our results that histological changes were improved in IM pregnant iNOS-KO mice infected with P. berghei NK65 (Fig. 2B-G). Moreover, Saa1 and IFN- $\gamma$ signalling pathway-related proteins, such as Gvin1 and Ifit3, were significantly lower in IM pregnant iNOS-KO mice infected with $P$. berghei NK65 than in IM pregnant WT mice infected with $P$. berghei NK65 (Table 3). However, Stat1 and Iigp1 levels in IM pregnant iNOS-KO mice infected with $P$. berghei NK65 were comparable to those in IM pregnant WT mice infected with $P$. berghei NK65, suggesting that IFN- $\gamma$ signalling was activated in IM pregnant iNOS-KO mice infected with $P$. berghei NK65. In addition, the levels of IFN- $\gamma$ and interleukin-10 in plasma were similar between IM pregnant WT and iNOS-KO mice infected with $P$. berghei NK65 (Fig. 3D, E).

By contrast, decreased levels of proteins, such as cytochrome $\mathrm{P} 450$ proteins (Table 2), in IM pregnant WT mice infected with $P$. berghei NK65 were improved in IM pregnant iNOS-KO mice infected with $P$. berghei NK65 (Table 4). These results indicate that nitric oxide produced by iNOS is involved in the development of liver dysfunction during malaria in pregnancy. 

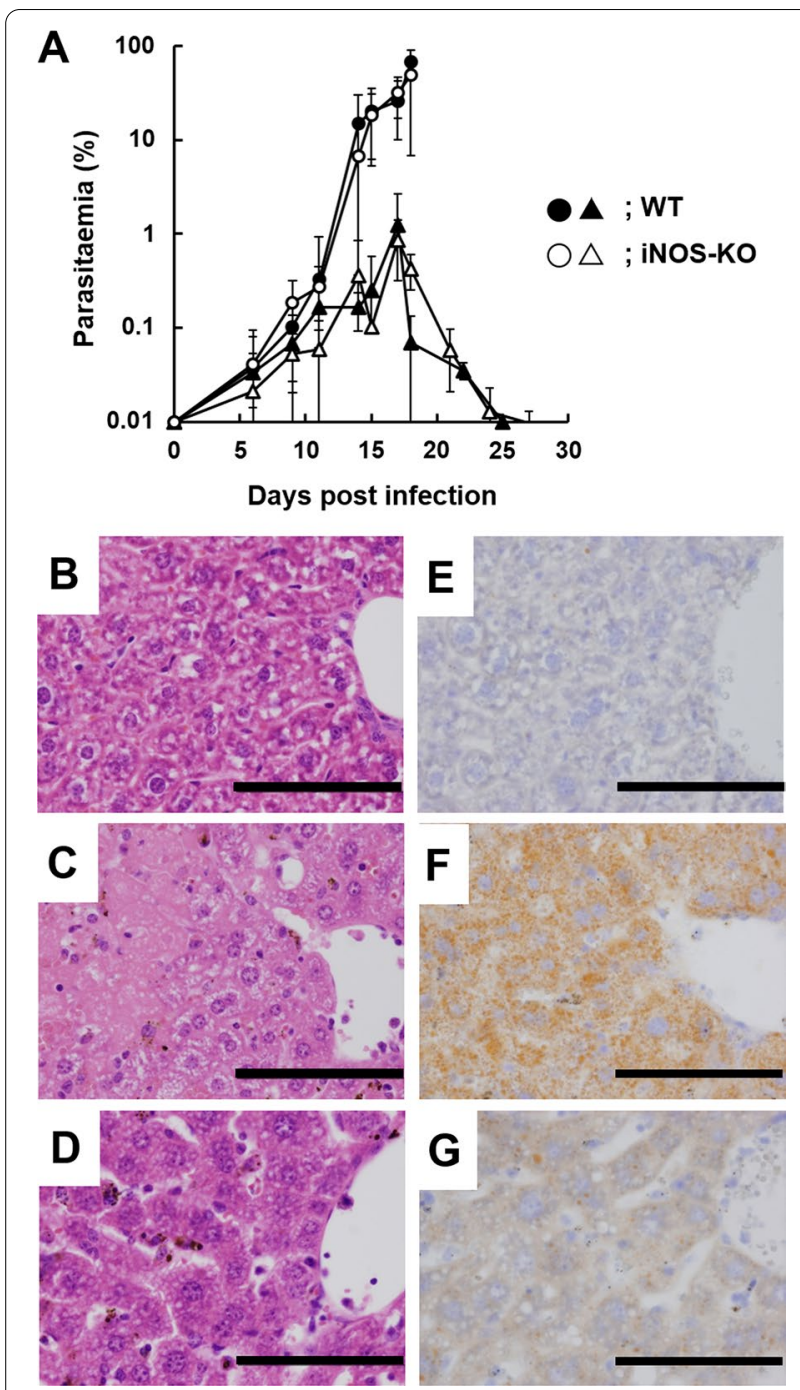

Fig. 2 Nitric oxide produced by inducible nitric oxide synthase (iNOS) is associated with microvesicular fatty infiltration. Immunized female wild-type (WT) and iNOS-knockout (KO) mice were infected intravenously with $1 \times 10^{4}$ erythrocytes infected with $P$. berghei NK65. A Course of parasitaemia. Closed and opened symbols indicate WT and INOS-KO mice, respectively. Circles and triangles indicate pregnant and non-pregnant mice, respectively. Results are shown as the means \pm standard deviation of three mice. The experiment was performed in triplicate with similar results. B-G Histological examination. Liver tissue staining with haematoxylin and eosin (B-D) and Sudan IV (E-G). Livers were obtained from mice on day 17 post-mating. B, E Immunized non-pregnant WT mice infected with $P$. berghei NK65. C, F Immunized pregnant WT mice infected with $P$. berghei NK65. D, G Immunized pregnant iNOS-KO mice infected with P. berghei NK65. Scale bar indicates $100 \mu \mathrm{m}$. Data are representative of six independent experiments

\section{Effect of iNOS deficiency on pregnancy outcomes}

Stillbirth was observed in IM pregnant WT and iNOSKO mice infected with $P$. berghei NK65. However, the pregnancy period of IM pregnant iNOS-KO mice infected with P. berghei NK65 was longer than that of IM pregnant WT mice infected with P. berghei NK65 (Fig. 4A). Accumulation of infected erythrocytes and a decreasing number of vascular branches were observed in the placentas of IM pregnant WT mice infected with $P$. berghei NK65 on days 16-18 post-mating, but not in IM pregnant iNOS-KO mice infected with $P$. berghei NK65 (Fig. 4B-G). These results suggest that nitric oxide produced by iNOS is involved in the enhancement of placental inflammation during malaria in pregnancy.

\section{Discussion}

Pregnant women infected with malaria parasites develop liver disease [8-10], but its pathogenesis remains unclear. Here, comparative proteomic analysis of the liver in a mouse model of malaria in pregnancy was performed. This analysis revealed that the levels of IFN- $\gamma$ signalling pathway-related proteins were increased in the livers of IM pregnant mice infected with $P$. berghei NK65. These results suggest that the inflammation induced by IFN- $\gamma$ signalling is involved in the development of liver disease during malaria in pregnancy.

In this study, the levels of Saa1 were increased in the liver of IM pregnant mice infected with P. berghei NK65. Increased levels of Saa have also been observed in plasma samples from HELLP patients [31]. Meanwhile, the levels of cytochrome $\mathrm{P} 450$ proteins, such as Cyp2a4, Сyp2c29, and Cyp2c70, were decreased in the liver during malaria in pregnancy. The levels of cytochrome $\mathrm{P} 450$ proteins are altered in patients with non-alcoholic fatty liver disease [32] and a corresponding animal model [33, 34]. These findings suggest that inflammation or infection affect levels of cytochrome $\mathrm{P} 450$ proteins in the liver.

iNOS-derived nitric oxide is thought to inhibit fatty acid oxidation. In fact, inhibition of $\beta$-oxidation is involved in the pathology of acetaminophen-induced hepatotoxicity [35]. However, iNOS-KO mice are resistant to hepatotoxicity caused by acetaminophen [36]. Decreased levels of mitochondrial and peroxisomal proteins were observed in the liver during malaria in pregnancy. While it is unclear whether iNOS-derived nitric oxide directly inhibits mitochondrial and peroxisomal proteins, results showing improvement of these protein levels by iNOS deficiency suggest that iNOSderived nitric oxide may be involved in mitochondrial or peroxisomal dysfunction in the liver during malaria in pregnancy.

Plasmodium berghei XAT is an attenuated parasite and mice infected with $P$. berghei XAT show low levels of parasitaemia and become cured spontaneously [37]. The course of parasitaemia and production of IFN- $\gamma$ are 

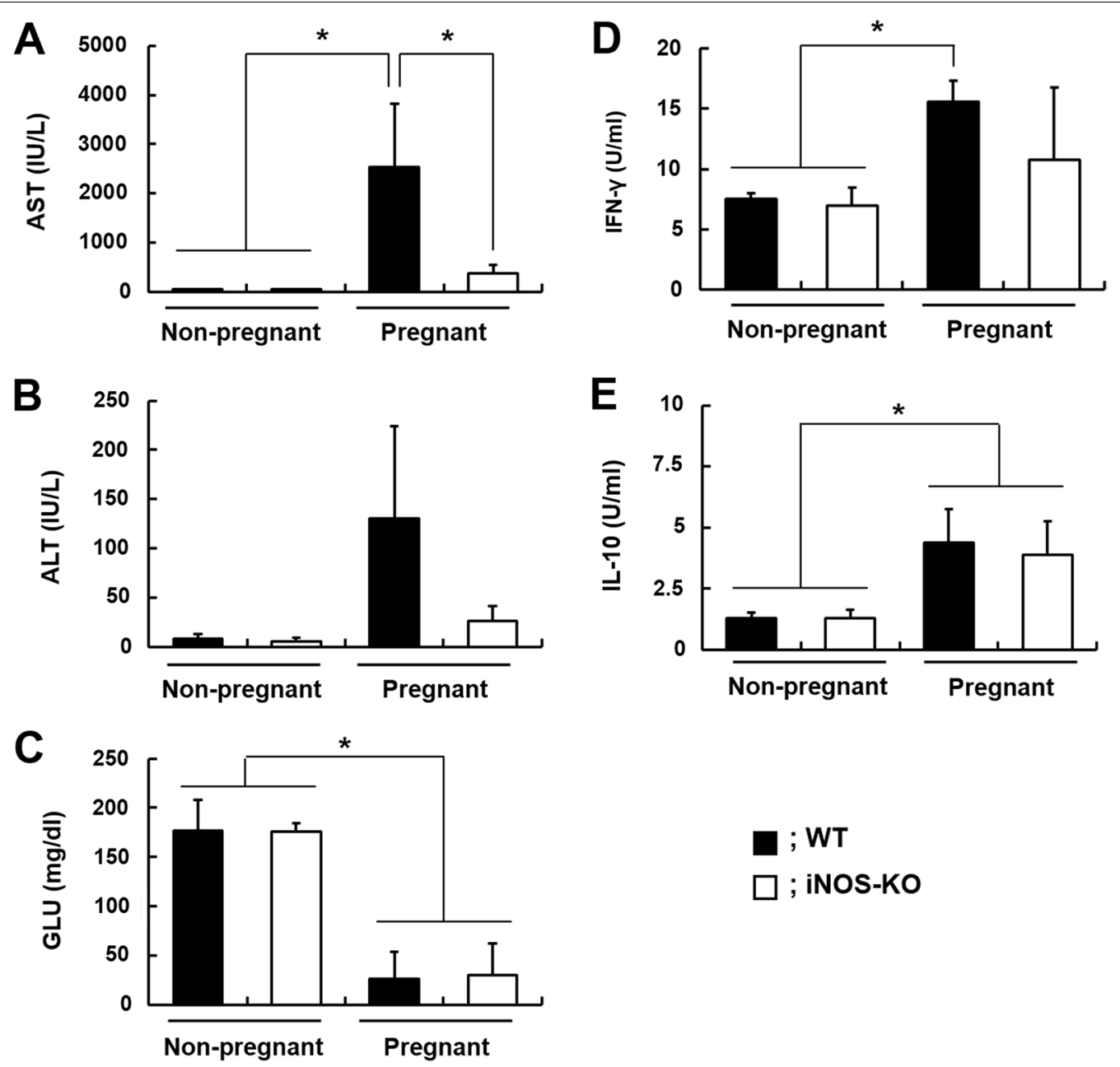

Fig. 3 Biochemical examination of blood and cytokine levels. Samples of blood were obtained from immunized pregnant and non-pregnant mice infected with P. berghei NK65 on day 17 post-mating. Plasma aspartic aminotransferase (AST) (A), alanine aminotransferase (ALT) (B), glucose (GLU) (C), interferon (IFN)- $\gamma(\mathbf{D})$, and interleukin (IL)-10 (E) levels are shown. Closed and opened symbols indicate wild-type (WT) and inducible nitric oxide synthase-knockout (iNOS-KO) mice, respectively. Non-pregnant indicate immunized non-pregnant WT and iNOS-KO mice infected with P. berghei NK65. Pregnant indicates immunized pregnant WT and iNOS-KO mice infected with P. berghei NK65. Asterisks indicate a statistically significant difference ( $P<0.05$ compared with immunized non-pregnant WT and iNOS-KO mice infected with $P$. berghei NK65; Tukey-Kramer and Dunnett test). Results are shown as the means \pm standard deviation of three mice. Experiments were performed in triplicate with similar results

not altered by iNOS deficiency [38]. In this study, when $P$. berghei XAT-immunized mice were infected with the lethal malaria parasite $P$. berghei NK65, their course of parasitaemia was not altered by iNOS deficiency. These findings indicate that acquisition of protective immunity by infection with malaria parasites is iNOS independent.

Liver disease associated with microvesicular fatty infiltration during malaria in pregnancy was improved by iNOS deficiency. It has been suggested that stress by alcohol or a high-cholesterol diet induce high levels of iNOS mRNA expression and cause fatty liver disease. These fatty liver diseases are improved by iNOS deficiency $[27,28]$, suggesting that iNOS plays a pivotal role in the development of liver disease. These results suggest that iNOS induced by inflammation or infection is an important mediator in liver disease during malaria in pregnancy, in addition to alcohol- or high-cholesterol diet-induced fatty liver disease.

In contrast to findings that nitric oxide produced by iNOS in inflammation is detrimental to organs, nitric oxide supplied by $\mathrm{L}$-arginine and the inhalation of nitric oxide were shown to have a protective/beneficial effect during severe malaria [39-42]. There are three isozymes that produce nitric oxide: nNOS (neuronal NOS, NOS1), iNOS (inducible NOS, NOS2), and eNOS (endothelial NOS, NOS3). eNOS plays an important role 
Table 3 Protein levels in the livers of immunized pregnant inducible nitric oxide synthase-knockout (iNOS-KO) mice infected with $P$. berghei NK65

\begin{tabular}{|c|c|c|c|}
\hline Description & Abbreviation & iNOS-KO & Accession \\
\hline Serum amyloid A-1 protein OS & Saa1 & 0.09 & P05366* \\
\hline Interferon-induced protein with tetratricopeptide repeats 3 OS & Ifit3 & 0.13 & Q64345* \\
\hline Metallothionein-2 OS & Mt2 & 0.27 & P02798* \\
\hline Ubiquitin-like protein ISG15 OS & $\operatorname{lsg} 15$ & 0.29 & Q64339* \\
\hline Ferritin light chain 1 OS & Ftl1 & 0.31 & P29391* \\
\hline T-cell-specific guanine nucleotide triphosphate-binding protein 1 OS & Tgtp1 & 0.32 & Q62293* \\
\hline Metallothionein-1 OS & Mt1 & 0.33 & P02802* \\
\hline Interferon-induced very large GTPase 1 OS & Gvin1 & 0.37 & Q80SU7* \\
\hline Perilipin-2 OS & Plin2 & 0.37 & P43883* \\
\hline Hemopexin OS & $\mathrm{Hpx}$ & 0.41 & Q91X72* \\
\hline UMP-CMP kinase 2, mitochondrial OS & Cmpk2 & 0.41 & Q3U5Q7* \\
\hline Haptoglobin OS & $\mathrm{Hp}$ & 0.42 & Q61646* \\
\hline Serine protease inhibitor A3K OS & Serpina3k & 0.44 & P07759* \\
\hline E3 ubiquitin-protein ligase RNF213 OS & Rnf213 & 0.46 & E9Q555* \\
\hline Apolipoprotein A-IV OS & Apoa4 & 0.47 & P06728* \\
\hline Metalloreductase STEAP4 OS & Steap4 & 0.47 & Q923B6* \\
\hline Galectin-3-binding protein OS & Lgals3bp & 0.49 & Q07797* \\
\hline Alpha-1-acid glycoprotein 8 OS & Orm8 & 0.50 & P21352* \\
\hline Coronin-1A OS & Corola & 0.51 & O89053 \\
\hline Pyruvate kinase PKM OS & Pkm & 0.55 & P52480 \\
\hline Fibrinogen gamma chain OS & Fgg & 0.62 & Q8VCM7 \\
\hline Signal transducer and activator of transcription 1 OS & Stat1 & 0.62 & P42225 \\
\hline Fibrinogen beta chain OS & $\mathrm{Fgb}$ & 0.63 & Q8K0E8 \\
\hline Neutrophilic granule protein OS & Ngp & 0.63 & O08692 \\
\hline Cytochrome P450 4A14 OS & Cyp4a14 & 0.63 & O35728 \\
\hline Fructose-bisphosphate aldolase A OS & Aldoa & 0.64 & P05064 \\
\hline Nicotinamide N-methyltransferase OS & Nnmt & 0.66 & O55239 \\
\hline Thymosin beta-4 OS & Tmsb4x & 0.67 & P20065 \\
\hline Glycogen [starch] synthase, liver OS & Gys2 & 0.68 & Q8VCB3 \\
\hline Dipeptidyl peptidase 1 OS & Ctsc & 0.70 & P97821 \\
\hline Intercellular adhesion molecule 1 OS & Icam 1 & 0.71 & P13597 \\
\hline Interferon-inducible GTPase 1 OS & ligp1 & 0.71 & Q9QZ85 \\
\hline Histone $\mathrm{H} 4$ OS & Hist1h4a & 0.73 & P62806 \\
\hline
\end{tabular}

Protein levels in the livers of immunized pregnant iNOS-KO mice infected with P. berghei NK65 compared with immunized pregnant wild-type (WT) mice infected with $P$. berghei NK65. Proteins with at least three peptide spectral matches and fold change $<0.735$ compared with immunized pregnant WT mice infected with $P$. berghei NK65 are listed. Asterisks indicate proteins with significantly lower levels than in immunized pregnant WT mice infected with $P$. berghei NK65 ( $<0.5$-fold change). Additional file 3: Table S2 shows all proteins detected in this study. Data are representative of three independent experiments

in maintaining endothelial homeostasis, such as angiogenesis and platelet production [43]. iNOS generates a larger amount of nitric oxide than eNOS or nNOS and is involved in cytotoxic functions [44]. L-Arginine supplementation and inhaled nitric oxide regulate angiopoietin levels and von Willebrand factor release [39-42]. These findings suggest that L-arginine and the inhalation of nitric oxide supply an appropriate amount of nitric oxide and play an auxiliary role alongside eNOS.

In this study, levels of the anti-inflammatory cytokine IL-10 in plasma were elevated in pregnant mice. During pregnancy, IL-10-producing regulatory $\mathrm{T}$ cells expand, conferring immune tolerance [45]. In IM pregnant mice infected with $P$. berghei NK65, this would suppress the acquired immunity against malaria parasites, thus increasing parasitaemia. The development of liver disease in IM pregnant WT mice infected with $P$. berghei NK65 suggested that the development of liver disease during malaria in pregnancy was not suppressed by IL-10. A previous study showed that liver damage during $P$. berghei NK65 induced infection was mediated by IL-27 in an IL-10 independent manner [46]. These findings 
Table 4 Upregulated proteins in the livers of immunized pregnant inducible nitric oxide synthase-knockout (iNOS-KO) mice infected with P. berghei NK65

\begin{tabular}{llll}
\hline Description & Abbreviation & iNOS-KO & Accession \\
\hline Cytochrome P450 1A2 OS & Cyp1a2 & 2.00 & P00186 \\
Glutathione S-transferase A2 OS & Gsta2 & 2.01 & P10648 \\
Sulfide:quinone oxidoreductase, mitochondrial OS & Sardl & 2.03 & Q9R112 \\
Carbonic anhydrase 3 OS & Ca3 & 2.08 & P16015 \\
Serum paraoxonase/arylesterase 1 OS & Pon1 & 2.11 & P52430 \\
L-gulonolactone oxidase OS & Gulo & 2.13 & P58710 \\
Cytochrome P450 2C29 OS & Cyp2c29 & 2.13 & Q64458 \\
Guanidinoacetate N-methyltransferase OS & Gamt & 2.16 & O35969 \\
Dimethylaniline monooxygenase [N-oxide-forming] 3 OS & Fmo3 & 2.22 & P97501 \\
Antithrombin-III OS & Serpinc1 & 2.24 & P32261 \\
Methyltransferase-like protein 7B OS & Mettl7b & 2.36 & Q9DD20 \\
Cytochrome P450 2A4 OS & Cyp2a4 & 2.66 & P15392 \\
Pericentrin OS & Pcnt & 2.66 & P48725 \\
Cytochrome P450 2C37 OS & Cyp2c37 & 3.19 & P56654 \\
Corticosteroid-binding globulin OS & Serpina6 & 3.91 & Q6XV770 \\
Cytochrome P450 2C54 OS & Cyp2c54 & 4.20 & Q91W64 \\
Cytochrome P450 2C70 OS & Cyp2c70 & & \\
\hline
\end{tabular}

Protein levels in the livers of immunized pregnant iNOS-KO mice infected with $P$. berghei NK65 compared with immunized pregnant WT mice infected with $P$. berghei NK65. Proteins with at least three peptide spectral matches and fold change $>2.0$ compared with immunized pregnant WT mice infected with $P$. berghei NK65 are listed. Additional file 3: Table S2 shows all proteins detected in this study. Data are representative of three independent experiments

point to a role for IL-27 in suppressing the development of liver disease during malaria in pregnancy. The elevated serum level of IL-27 in women with severe preeclampsia suggests IL-27 as a useful biomarker for disease severity in preeclampsia [47].

In IM iNOS-KO mice infected with $P$. berghei NK65, all infants were stillborn. These results suggest that nitric oxide produced by iNOS is not essential for foetal survival. However, pregnancy was longer in IM iNOSKO mice infected with $P$. berghei NK65 than in IM WT mice infected with $P$. berghei NK65. Placental pathology observed in IM pregnant WT mice infected with $P$. berghei NK65 was not observed in IM pregnant iNOSKO mice infected with $P$. berghei NK65. These results suggest that nitric oxide produced by iNOS may be involved in the enhancement of placental inflammation. Oxidative stress in placental mitochondria and peroxisomes may play a role in foetal fatty acid oxidation disorders during pregnancy $[48,49]$. Based on these findings, placental pathology may be associated with maternal liver diseases during malaria in pregnancy.

In contrast to IM pregnant mice infected with $P$. berghei NK65, liver disease in unimmunized pregnant mice infected with $P$. berghei NK65 was not improved by iNOS deficiency. $\mathrm{CD} 8^{+} \mathrm{T}$ cells have been shown to play a pivotal role in the development of liver disease in unimmunized mice infected with P. berghei NK65 [30]. From these findings, the contribution of iNOS is considered to be much less than that of $\mathrm{CD} 8^{+} \mathrm{T}$ cells in the development of liver disease in unimmunized mice infected with $P$. berghei NK65. By contrast, the immune response induced in IM pregnant mice infected with $P$. berghei NK65 may differ from that in unimmunized pregnant mice infected with $P$. berghei NK65 because immunized mice have acquired protective immunity against malaria parasites, suggesting that iNOS may play a role in maternal liver diseases in pregnant women living in malariaendemic areas.

In this study, alteration of the liver proteome during malaria in pregnancy was detected. Because differentiating HELLP-like syndrome during malaria in pregnancy can be challenging, the identification of biomarkers is necessary. The decreased levels of mitochondrial and peroxisomal proteins observed in this study are potential biomarkers of liver disease during malaria in pregnancy. Furthermore, a causative role of iNOS in liver disease associated with microvesicular fatty infiltration during malaria in pregnancy was demonstrated in this study. iNOS is known to be associated with metabolic diseases. In vivo models of liver disease during malaria in pregnancy will further our understanding of the role of the iNOS-mediated metabolic response and the pathogenesis of high-risk liver diseases of pregnancy, such as acute fatty liver. 


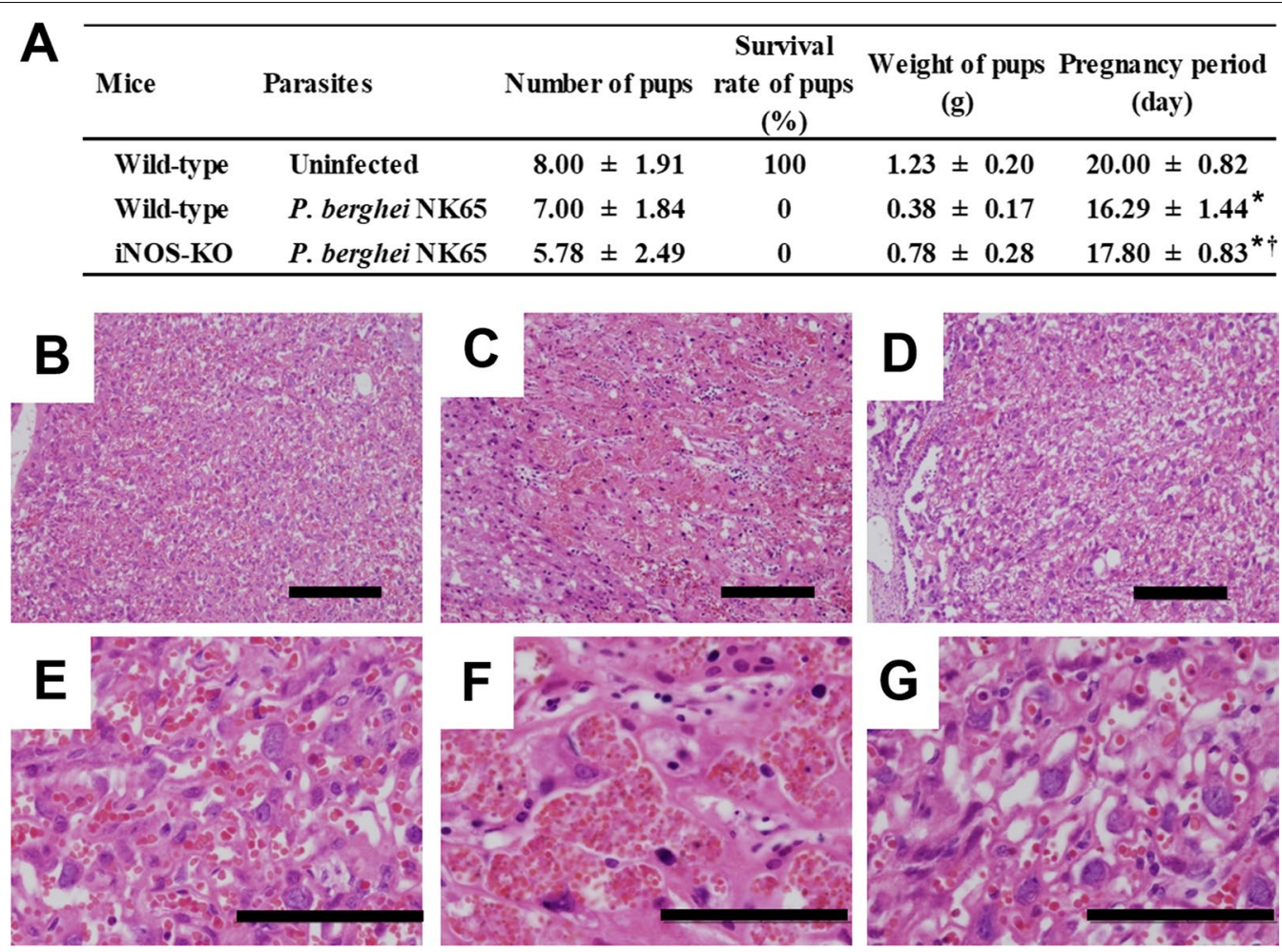

Fig. 4 Inducible nitric oxide synthase (iNOS) may be involved in adverse pregnancy outcomes during malaria. A Pregnancy outcomes in uninfected pregnant wild-type (WT) mice and immunized pregnant WT and iNOS-knockout (KO) mice infected with P. berghei NK65. Number, survival rate, and weight of fetuses were measured at delivery. Experiments using three mice were performed in triplicate with similar results. Asterisks indicate a significant difference compared with the pregnancy period of uninfected pregnant WT mice $(P<0.05$, Tukey-Kramer and Dunnett test). Dagger indicates a significant difference compared with the pregnancy period of immunized pregnant WT mice infected with P. berghei NK65. B-G Representative haematoxylin and eosin-stained placental sections are shown. Placentas were obtained from uninfected pregnant WT mice and immunized pregnant WT and iNOS-KO mice infected with P. berghei NK65 on day 17 post-mating. B-D Low magnification images of placentas. Scale bars indicate $200 \mu \mathrm{m}$. E-G Higher magnification of the labyrinth region. Scale bars indicate $100 \mu \mathrm{m}$. B, E Uninfected pregnant WT mice. C, F Immunized pregnant WT mice infected with P. berghei NK65. D, G Immunized pregnant iNOS-KO mice infected with P. berghei NK65. Data are representative of six independent experiments

\section{Conclusions}

iNOS plays a causative role in the development of liver disease during malaria in pregnancy.

\section{Abbreviations}

AFLP: Acute fatty liver of pregnancy; ALT: Alanine aminotransferase; AST: Aspartic aminotransferase; HELLP: Hemolytic anemia; elevated liver enzymes; low platelet count; iNOS: Inducible nitric oxide synthase; LCHAD: Long-chain 3-hydroxyacyl CoA dehydrogenase; NAFLD: Non-alcoholic fatty liver disease; PSMs: Peptide spectral matches.

\section{Supplementary Information}

The online version contains supplementary material available at https://doi. org/10.1186/s12936-021-03999-2.

Additional file 1: Table S1. Comparative proteomic analysis of livers of immunized pregnant mice infected with P. berghei NK65. Livers were obtained from unimmunized pregnant mice without infection (naïve pregnant mice) and unimmunized non-pregnant mice without infection (naïve virgin mice), and immunized pregnant and non-pregnant mice infected with P. berghei NK65 on day 17 post-mating. Proteins showing one or two peptide spectral matches were excluded. Protein levels were normalized to actin, cytoplasmic 1 (accession: P60710).
Additional file 2: Figure S1. Nitric oxide produced by inducible nitric oxide synthase (iNOS) is not involved in the development of liver injury during primary infection with lethal malaria parasites. Unimmunized wild-type (WT) and iNOS-knockout (KO) female mice were placed with male WT mice for 1 day. Mice on day 12 post-mating were infected with $1 \times 10^{4}$ erythrocytes infected with Plasmodium berghei NK65. (A) Course of parasitaemia. Closed and opened symbols indicate WT and iNOS-KO mice, respectively. Circles and triangles indicate pregnant and non-pregnant mice, respectively. Results are shown as the means \pm standard deviation of three mice. (B-E) Liver tissue staining with haematoxylin and eosin. Livers were obtained from mice on day 7 post-infection. (B) Unimmunized non-pregnant WT mice infected with P. berghei NK65. (C) Unimmunized pregnant WT mice infected with P. berghei NK65. (D) Unimmunized nonpregnant iNOS-KO mice infected with P. berghei NK65. (E) Unimmunized pregnant iNOS-KO mice infected with P. berghei NK65. Scale bar indicates $100 \mu \mathrm{m}$. Data are representative of three independent experiments.

Additional file 3: Table S2. Comparative proteomic analysis of livers of immunized pregnant inducible nitric oxide synthase-knockout (iNOS-KO) mice infected with $P$. berghei NK65. Livers were obtained from immunized pregnant mice infected with $P$. berghei NK65 on day 17 post-mating. Proteins showing one or two peptide spectral matches were excluded. Protein levels were normalized to actin, cytoplasmic 1 (accession: P60710). 


\section{Acknowledgements}

The English in this document has been checked by at least two professional editors, both native speakers of English. For a certificate, please see: http:// www.textcheck.com/certificate/vaNO28.

\section{Authors' contributions}

MN designed research; MN, TF and SM performed research; MN, TF, JM and FK analyzed data; and MN and FK wrote the paper. All authors read and approved the final manuscript.

\section{Funding}

This work was supported by a Grant-in-Aid for Scientific Research (C) from JSPS to M.N. (No. $18 \mathrm{~K} 07093$ and No. 21 K06997). The funders had no role in study design, data collection and analysis, decision to publish, or preparation of the manuscript.

\section{Availability of data and materials}

All data generated or analysed during this study are included in this published article and its additional files.

\section{Declarations}

\section{Ethics approval and consent to participate}

The experiments were approved (\#220) by the Experimental Animal Ethics Committee of Kyorin University School of Medicine, Tokyo, and all experiments were performed in accordance with guidelines and regulations of animal experimentation at Kyorin University. All animal experiments were also carried out in compliance with the ARRIVE guidelines.

\section{Consent for publication}

All contributing authors agreed consent for publication of the manuscript by Malaria Journal.

\section{Competing interests}

The authors declare that they have no competing interests.

\section{Author details}

'Department of Infectious Diseases, Kyorin University School of Medicine, 6-20-2 Shinkawa, Mitaka, Tokyo 181-8611, Japan. ${ }^{2}$ Department of Pharmacology and Toxicology, Kyorin University School of Medicine, Tokyo, Japan. ${ }^{3}$ Department of Molecular Pathology, Tokyo Medical University, Tokyo 1608402, Japan. ${ }^{4}$ Department of Environmental Science, School of Life and Environmental Science, Azabu University, Kanagawa 252-5201, Japan.

Received: 28 September 2021 Accepted: 1 December 2021

Published online: 14 December 2021

\section{References}

1. Menendez C. Malaria during pregnancy: a priority area of malaria research and control. Parasitol Today. 1995;11:178-83.

2. Nosten F, Rogerson SJ, Beeson JG, McGready R, Mutabingwa TK, Brabin B. Malaria in pregnancy and the endemicity spectrum: what can we learn? Trends Parasitol. 2004;20:425-32.

3. Duffy PE. Plasmodium in the placenta: parasites, parity, protection, prevention and possibly preeclampsia. Parasitology. 2007:134:1877-81.

4. Rogerson SJ, Boeuf P. New approaches to pathogenesis of malaria in pregnancy. Parasitology. 2007;134:1883-93.

5. Endeshaw Y. Malaria in pregnancy: clinical features and outcome of treatment. Ethiop Med J. 1991;29:103-8.

6. Steketee RW, Nahlen BL, Parise ME, Menendez C. The burden of malaria in pregnancy in malaria-endemic areas. Am J Trop Med Hyg. 2001;64:28-35.

7. Khan W, Zakai HA, Umm EA. Clinico-pathological studies of Plasmodium falciparum and Plasmodium vivax - malaria in India and Saudi Arabia. Acta Parasitol. 2014:59:206-12.

8. Tournoy J, Dapper I, Spitz B, Meersseman W, Van Wijngaerden E, Lagrou $K$, et al. Haemolysis, elevated liver enzymes, and thrombocytopenia in a 34-year-old pregnant woman. Lancet. 2006;368:90.
9. Ducarme G, Thuillier C, Wernet A, Bellier C, Luton D. Malaria in pregnant woman masquerading as HELLP syndrome. Am J Perinatol. 2010;27:171-2

10. Castillo Medina NM, Velázquez Fonseca J, Hernández Pacheco JA, Acevedo Tacuba JL. [Gestational malaria: HELLP syndrome mistaken diagnosis](in Spanish). Ginecol Obstet Mex. 2008;76:287-91.

11. Piñeros JG, Tobon-Castaño A, Alvarez G, Portilla C, Blair S. Maternal clinical findings in malaria in pregnancy in a region of northwestern Colombia. Am J Trop Med Hyg. 2013:89:520-6.

12. Mineo S, Niikura M, Inoue S, Kuroda M, Kobayashi F. Development of severe pathology in immunized pregnant mice challenged with lethal malaria parasites. Infect Immun. 2013;81:3865-71.

13. Liu J, Ghaziani TT, Wolf JL. Acute fatty liver disease of pregnancy: updates in pathogenesis, diagnosis, and management. Am J Gastroenterol. 2017;112:838-46

14. Ibdah JA. Acute fatty liver of pregnancy: an update on pathogenesis and clinical implications. World J Gastroenterol. 2006;12:7397-404.

15. Tran TT, Ahn J, Reau NS. ACG clinical guideline: liver disease and pregnancy. Am J Gastroenterol. 2016;111:176-94.

16. Nelson DB, Byrne JJ, Cunningham FG. Acute fatty liver of pregnancy. Obstet Gynecol. 2021;137:535-46.

17. Laubach VE, Shesely EG, Smithies O, Sherman PA. Mice lacking inducible nitric oxide synthase are not resistant to lipopolysaccharide-induced death. Proc Natl Acad Sci USA. 1995;92:10688-92.

18. Niikura M, Kamiya S, Kita K, Kobayashi F. Coinfection with nonlethal murine malaria parasites suppresses pathogenesis caused by Plasmodium berghei NK65. J Immunol. 2008;180:6877-84.

19. Niikura M, Fukutomi T, Fukui K, Inoue SI, Asahi H, Kobayashi F. G-strand binding protein 2 is involved in asexual and sexual development of Plasmodium berghei. Parasitol Int. 2020;76:102059.

20. Niikura M, Inoue SI, Fukutomi T, Yamagishi J, Asahi H, Kobayashi F. Comparative genomics and proteomic analyses between lethal and nonlethal strains of Plasmodium berghei. Exp Parasitol. 2018;185:1-9.

21. Wang G, Wu WW, Zhang Z, Masilamani S, Shen RF. Decoy methods for assessing false positives and false discovery rates in shotgun proteomics. Anal Chem. 2009:81:146-59.

22. Käll L, Canterbury JD, Weston J, Noble WS, MacCoss MJ. Semi-supervised learning for peptide identification from shotgun proteomics datasets. Nat Methods. 2007:4:923-5

23. Niikura M, Fukutomi T, Mineo S, Mitobe J, Kobayashi F. Malaria in the postpartum period causes damage to the mammary gland. PLOS ONE. 2021:16:e0258491.

24. Miyagami T, Igarshi I, Suzuki M. Plasmodium berghei: long lasting immunity induced by a permanent attenuated mutant. Zentralbl Bakteriol Mikrobiol Hyg A. 1987;264:502-12.

25. Tsai TH, Chen E, Li L, Saha P, Lee HJ, Huang LS, et al. The constitutive lipid droplet protein PLIN2 regulates autophagy in liver. Autophagy. 2017;13:1130-44

26. MacMicking JD. Interferon-inducible effector mechanisms in cell-autonomous immunity. Nat Rev Immunol. 2012;12:367-82.

27. McKim SE, Gäbele E, Isayama F, Lambert JC, Tucker LM, Wheeler MD, et al. Inducible nitric oxide synthase is required in alcohol-induced liver injury: studies with knockout mice. Gastroenterology. 2003;125:1834-44.

28. Anavi S, Eisenberg-Bord M, Hahn-Obercyger M, Genin O, Pines M, Tirosh $\mathrm{O}$. The role of iNOS in cholesterol-induced liver fibrosis. Lab Invest. 2015;95:914-24.

29. Spruss A, Kanuri G, Uebel K, Bischoff SC, Bergheim I. Role of the inducible nitric oxide synthase in the onset of fructose-induced steatosis in mice. Antioxid Redox Signal. 2011;14:2121-35.

30. Waki S, Uehara S, Kanbe K, Ono K, Suzuki M, Nariuchi H. The role of T cells in pathogenesis and protective immunity to murine malaria. Immunology. 1992;75:646-51.

31. Heitner JC, Koy C, Kreutzer M, Gerber B, Reimer T, Glocker MO. Differentiation of HELLP patients from healthy pregnant women by proteome analysis-on the way towards a clinical marker set. J Chromatogr B Analyt Technol Biomed Life Sci. 2006;840:10-9.

32. Fisher CD, Lickteig AJ, Augustine LM, Ranger-Moore J, Jackson JP, Ferguson SS, et al. Hepatic cytochrome P450 enzyme alterations in humans with progressive stages of nonalcoholic fatty liver disease. Drug Metab Dispos. 2009:37:2087-94. 
33. Chiba T, Noji K, Shinozaki S, Suzuki S, Umegaki K, Shimokado K. Dietinduced non-alcoholic fatty liver disease affects expression of major cytochrome P450 genes in a mouse model. J Pharm Pharmacol. 2016;68:1567-76.

34. Na AY, Jo JJ, Kwon OK, Shrestha R, Cho PJ, Kim KM, et al. Investigation of nonalcoholic fatty liver disease-induced drug metabolism by comparative global toxicoproteomics. Toxicol Appl Pharmacol. 2018;352:28-37.

35. Anavi S, Tirosh O. iNOS as a metabolic enzyme under stress conditions. Free Radic Biol Med. 2020;146:16-35.

36. Abdelmegeed MA, Jang S, Banerjee A, Hardwick JP, Song BJ. Robust protein nitration contributes to acetaminophen-induced mitochondrial dysfunction and acute liver injury. Free Radic Biol Med. 2013;60:211-22.

37. Waki S, Nakazawa S, Taverne J, Targett GA, Playfair JH. Immunity to an attenuated variant of Plasmodium berghei: role of some non-specific factors. Parasitology. 1985;91(Pt 2):263-72.

38. Yoneto T, Yoshimoto T, Wang CR, Takahama Y, Tsuji M, Waki S, et al. Gamma interferon production is critical for protective immunity to infection with blood-stage Plasmodium berghei XAT but neither NO production nor NK cell activation is critical. Infect Immun. 1999;67:2349-56.

39. McDonald CR, Cahill LS, Gamble JL. Malaria in pregnancy alters I-arginine bioavailability and placental vascular development. Sci Transl Med. 2018;10:eaan6007.

40. Ngai M, Weckman AM, Erice C, McDonald CR, Cahill LS, Sled JG, et al. Malaria in pregnancy and adverse birth outcomes: new mechanisms and therapeutic opportunities. Trends Parasitol. 2020;36:127-37.

41. Chua CLL, Hasang W, Rogerson SJ, Teo A. Poor birth outcomes in malaria in pregnancy: recent insights into mechanisms and prevention approaches. Front Immunol. 2021;12:621382.

42. Serghides L, Kim H, Lu Z, Kain DC, Miller C, Francis RC, et al. Inhaled nitric oxide reduces endothelial activation and parasite accumulation in the brain, and enhances survival in experimental cerebral malaria. PLoS ONE. 2011;6:e27714.

43. Heiss C, Rodriguez-Mateos A, Kelm M. Central role of eNOS in the maintenance of endothelial homeostasis. Antioxid Redox Signal. 2015;22:1230-42.

44. Papapetropoulos A, Rudic RD, Sessa WC. Molecular control of nitric oxide synthases in the cardiovascular system. Cardiovasc Res. 1999;43:509-20.

45. Jørgensen N, Persson G, Hviid TVF. The tolerogenic function of regulatory T cells in pregnancy and cancer. Front Immunol. 2019;10:911.

46. Findlay EG, Greig R, Stumhofer JS, Hafalla JC, de Souza JB, Saris CJ, et al. Essential role for IL-27 receptor signaling in prevention of Th1mediated immunopathology during malaria infection. J Immunol. 2010;185:2482-92.

47. Jahantigh D, Mousavi M, Forghani F, Javan MR, Movahedinia S, Rezaei M. Association between maternal circulating IL-27 levels and preeclampsia. Cytokine. 2018;102:163-7.

48. Shekhawat P, Bennett MJ, Sadovsky Y, Nelson DM, Rakheja D, Strauss AW. Human placenta metabolizes fatty acids: implications for fetal fatty acid oxidation disorders and maternal liver diseases. Am J Physiol Endocrinol Metab. 2003;284:E1098-105.

49. Natarajan SK, Thangaraj KR, Eapen CE, Ramachandran A, Mukhopadhya A Mathai $M$, et al. Liver injury in acute fatty liver of pregnancy: possible link to placental mitochondrial dysfunction and oxidative stress. Hepatology. 2010;51:191-200

\section{Publisher's Note}

Springer Nature remains neutral with regard to jurisdictional claims in published maps and institutional affiliations.

Ready to submit your research? Choose BMC and benefit from:

- fast, convenient online submission

- thorough peer review by experienced researchers in your field

- rapid publication on acceptance

- support for research data, including large and complex data types

- gold Open Access which fosters wider collaboration and increased citations

- maximum visibility for your research: over $100 \mathrm{M}$ website views per year

At $\mathrm{BMC}$, research is always in progress.

Learn more biomedcentral.com/submissions 